\title{
The effect of intrauterine insemination time on semen parameters
}

\author{
Elvan Koyun, Recep Emre Okyay, Ömer Erbil Doğan, Müge Kovalı, Sultan Seda Doğan, Bülent Gülekli \\ Department of Obstetrics and Gynaecology, Dokuz Eylül University Faculty of Medicine, Izmir, Turkey
}

\section{Abstract}

Objective: The purpose of this observational study was to determine whether semen parameters (concentration, motility) were affected by the interval between the onset of postwash sperm incubation and intrauterine insemination (IUI) time.

Material and Methods: Semen specimens of 100 normozoospermic men collected at the clinic were allowed 20 minutes for liquefaction at room temperature. Semen samples were subjected to both macroscopic and microscopic examinations. After centrifugation in a density gradient column and sperm-washing medium, the samples were kept in an incubator. After 30 minutes, 60 minutes, and 120 minutes, the concentration and motility were recorded.

Results: According the results of the Bonferroni post hoc test, there were significant differences in values of mean sperm count, percent progressive sperm motility, and total motile sperm count between 30 minutes and 120 minutes $(p=0.000, p=0.000$, and $p=0.000)$ and between 60 minutes and 120 minutes $(p=0.000, p=0.000$, and $p=0.001)$, but there was no significant difference between 30 minutes and 60 minutes ( $p=1$, $\mathrm{p}=0.173$, and $\mathrm{p}=1$ ).

Conclusion: This study demonstrated that sperm parameters are negatively affected from prolonged incubation time. A maximum 60-minute limit of the interval between the onset of postwash sperm incubation and IUI time may increase pregnancy rates. (J Turk Ger Gynecol Assoc 2014; 15: 82-5)

Key words: IUI time, sperm wash, semen parameters

Received: 23 October, 2013

Accepted: 16 December, 2013

\section{Introduction}

Intrauterine insemination (IUI) is a method that has been used for infertile couples in fertility treatment for many years. IUI is the first referenced assisted reproductive technique (ART) for mild to moderate male infertility. IUI is noninvasive and very simple, and it is less expensive than classical in vitro fertilization (IVF) and intracytoplasmic injection (ICSI). IUI success depends on many factors such as drugs, the timing and number of cycles, and total motile sperm count after washing. Another important factor affecting the success of IUI is the amount of motile sperm that is inserted into the uterus (1). Most studies have suggested that the success of IUI therefore decreases in pregnancy rates if there is not a sufficient number of motile sperm after washing (2-5).

A semen analysis is the first step to accurately diagnosing male infertility. Sperm count, percentage of moving sperm (sperm motility), and the percentage of sperm with normal morphology are the main criteria for the quality of semen. Determining sperm quality often uses values established by the World Health Organization (WHO) 2010 (6). However, the cut-off values for total progressive motile sperm count are still controversial and vary between $0.3 \times 10^{6}$ and $20 \times 10^{6}(7)$.
The intervals between semen collection and IUI time could affect the sperm parameters and clinical pregnancy rates negatively. However, there are few studies on this issue (8). According to WHO criteria (2010), the interval between semen collection and giving it to the laboratory should not exceed 60 minutes (6). But, a few studies suggest that this interval should be shorter (8).

In this study, we aimed to determine the ideal interval between the onset of postwash sperm incubation and IUI time to increase IUI success.

\section{Material and Methods}

\section{Subjects}

In this study, 100 normozoospermic men underwent semen analysis at the Dokuz Eylül University IVF Unit. The mean age of the men was 32.7 \pm 5.2 years (range $23-47$ ).

Semen samples were obtained by masturbation after sexual abstinence periods (from 2 to 6 days). All semen specimens collected at the clinic were allowed 20 minutes for liquefaction at room temperature.

After liquefaction, the semen variables that were considered were ejaculate volume, sperm count, total motility, and total progressive motility (TPM). Standard semen parameters were analyzed according to the $2010 \mathrm{WHO}$ guidelines (6). 
Over 15 million sperm per milliliter of semen was considered normal. The lower reference limit for total sperm number was $39 \times 10^{6}$ spermatozoa per ejaculate. Total sperm count was calculated by multiplying semen volume by sperm concentration. Spermatozoa were categorized by the following different motility grades: 1) rapid progressive motility, 2) slow progressive motility, 3) nonprogressive motility, and 4) immotile sperm.

Total motility (normal $\geq 40 \%$ ) includes rapid progressive motility, slow progressive, and nonprogressive motility. Progressive motility (normal $\geq 32 \%$ ) includes rapid and slow progressive motility. Absolute values for total progressive motile spermatozoa count per ejaculate were also calculated by multiplying the percentage of total progressive sperm motility $(a+b)$ by sperm concentration per milliliter and by the volume (in milliliters) of the single ejaculate.

Morphology was evaluated by the Spermac staining procedure (FertiPro, Beernem, Belgium). The smears were stained with Spermac, and 200 spermatozoa were evaluated for morphology. These were abnormal acrosome, head, mid-piece, and tail forms. The smears were evaluated under a light microscope at $\times 1000$ magnification according to the method described by Kruger. The percentage of normal morphology in a sample was $4 \%$. If it was below this percentage, this condition was diagnosed as teratozoospermia (6).The ejaculation time, the beginning of sperm wash time, and the onset of incubation were recorded.

Semen samples were processed using density gradient medium (PureSperm ${ }^{\circledR}$, Nidacon, International AB, Sweden) and sperm wash medium (Sperm Wash, Nidacon, International AB, Sweden). For the experiment, we used PureSperm gradients of $90 \%$ and $50 \%$. All procedures were conducted under sterile conditions. Media that was used for the processes was brought to $37^{\circ} \mathrm{C}$. For the lower layer, PureSperm gradient $90 \%$ was transferred into a conical centrifuge tube using a sterile $1 \mathrm{~mL}$

Table 1. The baseline characteristics of prewash semen samples

\begin{tabular}{|l|c|}
\hline Semen parameter & Median value (IQR)* \\
\hline Semen volume $(\mathrm{mL})$ & $3(3)$ \\
\hline Sperm count $\left(10^{6} / \mathrm{mL}\right)$ & $60(41.5)$ \\
\hline Total progressive motility (\%) & $50(10)$ \\
\hline Total motility (\%) & $60(10)$ \\
\hline Morphologic normal form (\%) & $4(1)$ \\
\hline $\begin{array}{l}\text { *Data are presented as median } \\
\text { IQR: interquartile range }\end{array}$ \\
\hline
\end{tabular}

pipette. For the upper layer, PureSperm gradient 50\% was gently dispensed on top of the lower layer using a new sterile $1 \mathrm{~mL}$ pipette. Liquefied semen sample were then placed on top of the upper layer, and the tube was centrifuged for 15 minutes at $1800 \mathrm{rpm}$. The upper and lower layers were carefully aspirated without disturbing the pellet. Sperm wash medium was added, and the pellet was re-suspended using a $4 \mathrm{~mL}$ transfer pipette; then, the tube was centrifuged for 5 minutes at $1500 \mathrm{rpm}$. The supernatant was then removed, and $0.3-0.5 \mathrm{~mL}$ of sperm wash medium was dispensed on the top of the pellet gently. The tube was then incubated, and after 30 minutes, 60 minutes, and 120 minutes, the concentration and motility were recorded.

Ethics committee approval was received for the study, and all patients who participated in this study gave written informed consent forms.

\section{Statistical Analysis}

Statistical analysis of the data was done by SPSS software (version 16, SPSS, Chicago, USA). The data were analyzed by general linear models. A $P$ value $<0.05$ was considered statistically significant.

\section{Results}

The median age of males was 32 years (range 23-47). The median ejaculatory abstinence interval was 4 days (range 2-5). The baseline characteristics of prewash semen samples are shown in Table 1.

According to Table 2, values for mean sperm count and total motile sperm count after 60 minutes of incubation were the highest and the lowest after 120 minutes. The mean total percent progressive sperm motility after 30 minutes was the highest and the lowest after 120 minutes. It was found that when using the ANOVA test with repeated measures with a Greenhouse-Geisser correction, the mean scores for sperm concentration $(\mathrm{mil} / \mathrm{mL})$, percent progressive sperm motility (\%), and total motile sperm count (mil) were significantly different between groups $(\mathrm{F}=13.065, \mathrm{p}<0.0005, \mathrm{~F}=20.128, \mathrm{p}<0.0005$, $\mathrm{F}=11.454, \mathrm{p}<0.0005)$.

According the results of the Bonferroni post hoc test, there were significant differences in values of mean sperm count, percent progressive sperm motility, and total motile sperm count between 30 minutes and 120 minutes $(p=0.000, p=0.000$, and $\mathrm{p}=0.000)$ and between 60 minutes and 120 minutes $(\mathrm{p}=0.000$, $p=0.000$, and $p=0.001$ ), but there was no significant difference between 30 minutes and 60 minutes $(p=1, p=0.173, p=1)$.

Table 2. Marginal means (mean \pm SD) of postwash semen parameters in different periods

\begin{tabular}{|l|c|c|c|c|c|}
\hline \multirow{2}{*}{ Semen parameters (mean \pm min SD) } & \multicolumn{3}{|c|}{ Groups } \\
\cline { 2 - 6 } & $\mathbf{3 0} \mathbf{~ m i n}$ & $\mathbf{6 0}$ min & $\mathbf{1 2 0}$ min & F value \\
\hline Sperm count $\left(\times 10^{6} / \mathrm{mL}\right)$ & $30.2 \pm 25.5$ & $30.8 \pm 29.0$ & $21.5 \pm 19.1$ & 13.065 & 0.000 \\
\hline Total progressive motility $(\%)$ & $96.8 \pm 8.3$ & $95.2 \pm 10.4$ & $87.5 \pm 21.9$ & 20.128 & 0.000 \\
\hline Total motile sperm count $\left(\times 10^{6}\right)$ & $8.8 \pm 7.5$ & $9.2 \pm 10.9$ & $5.4 \pm 4.8$ & 11.454 & 0.000 \\
\hline
\end{tabular}




\section{Discussion}

Among the ARTs, IUI is considered a first-line procedure. IUI is simple, easy, and cheap, and it has the absence of potentially serious complications. Prior to an IUI, the semen to be used during the procedure must be prepared; this procedure is commonly referred to as sperm washing. According to the 2010 WHO guidelines, semen samples should be collected in a private room near the andrology laboratory, because freshness of the samples could affect fertility (6). Within minutes after ejaculation, the number of living sperm cells and their activity begin to drop off. Semen is affected from temperature changes and prolonged time (9-12). A freshly ejaculated semen sample might resume an admissible sperm progressive motility for up to 12 hours, and sperm cells will survive up to 24-48 hours (11, 12). Analysis of sperm should be conducted in a laboratory within 1 hour of collection according to the 2010 WHO guidelines (6).

Consumption of energy sources in the sperm-washing medium by the motile spermatozoa during a prolonged sperm washIUI interval could affect fertilization negatively, because sperm motility requires energy sources, such as glucose and fructose, and washed motile spermatozoa that are deprived of energy sources during a prolonged sperm wash-IUI interval might not be able to reach the fertilization site in the fallopian tubes after IUI. Another such factor could be premature (in vitro) capacitation of washed motile spermatozoa in the sperm-washing medium during a prolonged sperm wash-IUI interval, because seminal decapacitation factors are removed along with seminal plasma during the sperm wash procedure $(8,13)$.

Shimuzu et al. (14) published a study in 2009 to assess the relationship between the time interval from semen collection to sperm wash and IUI outcome; 1054 IUI treatment cycles were analyzed. The time interval from semen collection to sperm wash was divided into three groups: a) less than 3 hours, b) 3-5 hours, and c) over 5 hours. Total pregnancy rate was 14\%, and IUI pregnancy rate was not different between groups. Another study was done by Alexander et al. (15) to correlate pregnancy outcomes with the subject time intervals in 210 clomiphene citrate (CC)-IUI cycles. Mean intervals from collection to sperm washing, from sperm washing to IUI, and from collection time to IUI were similar in pregnant and non-pregnant CC-treated women. A similar study conducted in 2003 analyzed 132 IUI cycles to determine if IUI outcome was affected by place of semen collection (home or clinic) and intervals from semen collection to sperm wash, from sperm wash to IUI time, and from semen collection to IUI time. Yavas et al. (16) concluded that $\mathrm{CC}$ and human menopausal gonadotropin (hMG) result in similar pregnancy rates when semen is collected either at home or at the clinic. Pregnancy is not affected by intervals of semen collection-sperm wash, sperm wash-IUI, and semen collectionIUI in CC-treated women but is associated with shorter intervals in hMG-treated women. Yavaş and Selub (8) published another study in 2004. In this study, it was found that semen collection at the clinic resulted in a higher pregnancy rate than collection at home in hMG-treated but not in CC-treated women. Intervals of semen collection-sperm wash, sperm wash-IUI, and semen collection-IUI were shorter in pregnant than in nonpregnant hMG-treated women. Semen washed within 30 minutes after collection resulted in a higher pregnancy rate than that processed 31-60 minutes after collection in hMG-treated but not in CC-treated women. IUI performed within 90 minutes after collection resulted in a higher pregnancy rate than IUI performed at 91-120 minutes or >120 minutes after collection in hMGtreated but not in CC-treated women (8).

In a recent study, it was found that if washed sperm can be incubated for a minimum of 30 minutes at $37^{\circ} \mathrm{C}$, the pregnancy rate is optimum. The authors concluded that a longer period up to 180 minutes does not compromise the pregnancy rate (17). Different results in the literature could be based on the different techniques used to prepare the spermatozoa for IUI or technicians in the andrology laboratory. In our study, all semen specimens were collected at the clinic in private rooms near the andrology laboratory. All semen analyses and sperm wash procedures were performed by the same investigator (E.K.). There were no data related to IUI outcomes, because IUI was not performed on women. In this study, normozoospermic semen samples of 100 men who applied to our laboratory for sperm testing were analyzed.

In this study, independent from woman factor and thus from the hormone preparations used, we aimed to see whether semen parameters (concentration, motility) were affected from the interval between the onset of postwash sperm incubation and IUI time. We set a fixed duration for the liquefaction (20 minutes) and sperm wash procedure (25 minutes).

According to statistical data, sperm parameters are not affected adversely by the start of the washing process of semen samples followed by a fixed semen liquefaction time of 20 minutes and by the extension of the insemination period up to $60 \mathrm{~min}$ utes, on the condition of appropriate incubation of the semen sample. Nevertheless, as this period reached 120 minutes, we observed a significant decline in number of sperm, total progressive sperm percentage, and total motile sperm averages.

As a result, especially considering the human chorionic gonadotropin (hCG) injection time at busy centers, this period, with the condition of not exceeding 60 minutes from the beginning of the incubation up to the time of insemination, probably affects pregnancy rates positively. It is also aimed to review the pregnancy results by repeating this study on IUI patients with their recorded incubation start times and IUI times.

Ethics Committee Approval: Ethics commitee approval was received for this study.

Informed Consent: Written informed consent was obtained from patients who participated in this study.

Peer-review: Externally peer-reviewed.

Author contributions: Concept - E.K., R.E.O., O.E.D. ; Design - E.K., M.K., S.D.; Supervision - E.K., B.G. ; Resource - E.K., O.E.D., R.E.O., B.G. ; Materials - E.K., O.E.D., R.E.O., B.G.; Data Collection\&/or Processing E.K.; Analysis\&/or Interpretation - E.K.; Literature Search - E.K.; Writing -E.K.; Critical Reviews - E.K.

Conflict of Interest: No conflict of interest was declared by the authors. Financial Disclosure: The authors declared that this study has received no financial support. 


\section{References}

1. İltemir Duvan C, Berker B, Bayrak O, Aydos K, Özturk Turhan N, Şatıroğlu H. Comparison of semen parameters between pregnant and non-pregnant couples with male factor infertility during intrauterine insemination. Turk J Med Sci 2009; 39: 531-6.

2. Kılıçdağ EB, Bagış T, Haydardedeoğlu B, Tarım E, Aslan E, Erkanlı S, et al. The prognostic factors that could be effect pregnancy rates in intra uterine insemination (IUI) cycles. J Turk Soc Obstet Gynecol 2005; $2: 223-8$.

3. Nikbakht R, Saharkhiz N. The Influence of sperm morphology, total motile sperm count of semen and the number of motile sperm inseminated in sperm samples on the success of intrauterine insemination. Int J Fertil Steril 2011; 5: 168-73.

4. Alıcı B, Özkara H, Önal B, Akkuş E, Hattat H. The effect of total motile sperm count to the success of intrauterine insemination. Cerrahpaşa J Med 2000; 31: 61-5.

5. Miller DC, Hollenbeck BK, Smith GD, Randolph JF, Christman GM, Smith YR, et al. Processed total motile sperm count correlates with pregnancy outcome after intrauterine insemination. Urology 2002; 60: 497-501. [CrossRef]

6. World Health Organization. WHO manual for the examination of human semen and cervical mucus interaction. WHO Press; 2010.

7. Van Weert J-M, Repping S, Von Voorhis BJ, Bossuyt PMM, Mol BWJ. Performance of the postwash total motile sperm count as a predictor of pregnancy at the time of intrauterine insemination: a meta-analysis. Fertil Steril 2004; 82: 612-20. [CrossRef]

8. Yavas Y, Selub MR. Intrauterine insemination (IUI) pregnancy outcome is enhanced by shorter intervals from semen collection to sperm wash, from sperm wash to IUI time, and from semen collection to IUI time. Fertil Steril 2004; 82: 1638-47. [CrossRef]
9. Warikoo PK. Spermatogenesis, Sperm Maturation \& Sperm-oocyte Interaction in Human. In: Allahbadia GN, editor. Intrauterine Insemination. New Delhi: Jaypee Medical Publishers; 2006. p. 3-24.

10. Pasqualotto EB, Agarwal A. Effect of Male Factor on Pregnancy Outcome in Patients Undergoing IUI. In: Allahbadia GN, editor. Intrauterine Insemination. New Delhi: Jaypee Medical Publishers; 2006. p. 117-27.

11. Krause W, Bock K. Computer Assisted Sperm Analysis (CASA) in an Insemination Programme. In: Allahbadia GN, editor. Intrauterine Insemination. New Delhi: Jaypee Medical Publishers; 2006. p. 12839.

12. Gangrade BK. Modulation of Sperm Motility-use of Motility Enhancing Agents in Artificial Insemination. In: Allahbadia GN, editor. Intrauterine Insemination. New Delhi: Jaypee Medical Publishers; 2006. p. 149-57.

13. Gautam AN. Does the time interval between semen collection, processing \& insemination affect results of IUI? J Obstet Gynecol India; 2009: 59: 407-9.

14. Shimuzu Y, Yorimitsu T, Motoyama H, Ohara M, Kawamura T. Relationship between the time interval from semen collection to sperm wash and IUI outcome Fertil Steril 2009; 92: 145. [CrossRef]

15. Alexander CJ, King J, Lipari C, Zhao Y, Wallach EE. The effect of time intervals between semen collection, processing and intrauterine insemination (IUI) on pregnancy rates in clomiphene-citrateIUI cycles. Fertil Steril 2005; 84: 279. [CrossRef]

16. Yavas Y, Selubn MR, Lefkow H, Carmichael D. Intra-uterine insemination (IUI) outcome is enhanced by shorter intervals from semen collection to sperm wash, from sperm wash to IUI time, and from semen collection to IUI time. Fertil Steril 2003; 80: 152-3. [CrossRef]

17. Kılıçdağ EB. The effect of intervals from sperm wash to intra uterine insemination (IUI) time on pregnancy rate. J Turk Soc Obstet Gynecol 2012; 9: 159-63. [CrossRef] 